\title{
Influence of marital status on the survival of adults with extrahepatic/intrahepatic cholangiocarcinoma
}

\author{
Zhiqiang Chen ${ }^{1, *}$, Liyong Pu ${ }^{1, *}$, Wen Gao ${ }^{2, *}$, Long Zhang ${ }^{1}$, Guoyong Han ${ }^{1}$, Qin Zhu ${ }^{1}$, \\ Xiangcheng $\mathrm{Li}^{1}$, Jindao $\mathrm{Wu}^{1}$, Xuehao Wang ${ }^{1}$ \\ ${ }^{1}$ Department of Liver Surgery, The First Affiliated Hospital of Nanjing Medical University, Key Laboratory on Living Donor \\ Liver Transplantation, National Health and Family Planning Commission, Nanjing, Jiangsu Province, China \\ ${ }^{2}$ Department of Oncology, The First Affiliated Hospital of Nanjing Medical University, Nanjing, Jiangsu Province, China \\ "These authors contributed equally to this work
}

Correspondence to: Xuehao Wang, email: wangxh@njmu.edu.cn

Jindao Wu, email: wujindao@njmu.edu.cn

Xiangcheng Li, email: drxcli@njmu.edu.cn

Keywords: cholangiocarcinoma, marital status, SEER, survival analysis, prognosis

Received: December 08, $2016 \quad$ Accepted: February 28, $2017 \quad$ Published: March 17, 2017

Copyright: Chen et al. This is an open-access article distributed under the terms of the Creative Commons Attribution License (CC-BY), which permits unrestricted use, distribution, and reproduction in any medium, provided the original author and source are credited.

\section{ABSTRACT}

Although the prognostic value of marital status has been implicated in many cancers, its prognostic impact on cholangiocarcinoma has not yet been determined. The aim of this study was to examine the association between marital status and cholangiocarcinoma survival. We included 8,776 extrahepatic cholangiocarcinoma cases and 1,352 intrahepatic cholangiocarcinoma cases between 1973 and 2013 from the Surveillance, Epidemiology, and End Results database. We found widowed patients were more likely to be female, aged more than 70, and from low income areas. Multivariate analysis indicated that marital status was an independent prognostic factor for extrahepatic cholangiocarcinoma patients. Subgroup analysis suggested the widowed status independently predicted poor survival at regional stage and in older patients with intrahepatic cholangiocarcinoma. To conclude, marital status is a valuable prognostic factor in cholangiocarcinoma, and widowed patients are at greater risk of death than others.

\section{INTRODUCTION}

Cholangiocarcinoma, the second most common primary hepatic cancer after hepatocellular carcinoma (HCC), accounts for approximately $3 \%$ of all gastrointestinal malignancies $[1,2]$. Cholangiocarcinoma are anatomically classified as extrahepatic cholangiocarcinoma (ECC) or intrahepatic cholangiocarcinoma (ICC) according to its location with regard to the liver [3]. The incidence of ECC/ ICC has increased substantially in the past decades, and the newly diagnosed cases have exceeded 7,000 annually [4, 5]. Both ECC and ICC are highly lethal and characterized by an aggressive behavior with early lymphatic spread and distant metastasis. Total cholangiocarcinoma mortality for those aged more than 25 increased 36\% between 1999 and 2014, from 2.2 per 100,000 to 3.0 per $100,000[6,7]$.

An emerging number of studies have demonstrated the involvement of marriage in the clinical prognosis of various digestive system malignancies. Among pancreatic cancer patients, marital status was an independent prognostic factor
[8]. In patients with colorectal neuroendocrine neoplasms, the widowed group were at greater risk of cancer specific mortality [9]. Reportedly, widowed patients suffered from the poorest 5-year cancer specific survival in HCC [10]. In primary liver cancer patients, widowed patients had a survival disadvantage while married persons enjoyed survival benefits in both cancer-specific survival and overall survival [11]. Paradoxically, a cross-sectional study conducted in wetland communities of Ubon Ratchathani in Thailand implied that married participants had a 2.61 times higher risk of cholangiocarcinoma than unmarried participants [12].

In order to clarify the prognostic significance of marital status in cholangiocarcinoma, we performed a comprehensive population-based analysis. The data were obtained from the Surveillance, Epidemiology, and the End Results (SEER) cancer registry, which comprised about $97 \%$ of incident cancer cases from 17 cancer registries representing $28 \%$ of the US population. Due to the differences in pathogenesis, etiologic risk factors, and 
genetic characteristics between ECC and ICC [13-18], ECC cause-specific survival (ECSS) and ICC cause-specific survival (ICSS) were examined separately in our analysis.

\section{RESULTS}

\section{Baseline patient characteristics}

A total of 10,128 eligible cholangiocarcinoma patients were included during the 40-year study period (from 1973 to 2013), comprising 5,265 male and 4,863 female patients. Among these patients, 5,926 (58.5\%) were married, $1,150(11.4 \%)$ had never married, 947 (9.4\%) were divorced/separated and 2,105 (20.8\%) were widowed. Significant differences were observed in all subgroups, including gender, age, ethnicity, year of diagnosis, pathological grading, TNM stage, SEER stage and socioeconomic status. All comparisons were statistically significant $(P<0.001)$. Of note, married patients were more likely to be male $(63.2 \%)$, while widowed patients have the highest proportion $(79.4 \%)$ of female patients. Widowed patients also had a greater proportion $(82.5 \%)$ of older patients. Additionally, the socioeconomic status of married individuals was better than other unmarried individuals. Married patients had the largest proportion of low poverty $(16.3 \%)$, and suffered the least from high poverty (15.4\%). The baseline cholangiocarcinoma patient demographics and malignancy characteristics were summarized in Table 1.

After further analyzing these differences in ECC and ICC respectively, as shown in Supplementary Table 1 and Supplementary Table 2, these differences were also observed in ECC patients. In ICC patients, by contrast, only gender, age, ethnicity, TNM stage and SEER stage remained significant.

\section{Influence of marital status on ECSS}

Married individuals had a better 5-year ECSS than unmarried individuals $(13.9 \%$ vs $10.1 \%)(P<0.001)$ (Figure 1A). Specifically, the 5-year ECSS was $13.4 \%$ in the never married group, $10.2 \%$ in the divorced/separated group and $8.4 \%$ in the widowed group (Figure 1B). Female $(P<0.001)$, older age $(P<0.001)$, black race $(P=0.005)$, the latest year of diagnosis $(P<0.001)$, poor/ anaplastic pathological grading $(P<0.001)$, TNM stage III/ IV $(P<0.001)$, SEER distant stage $(P<0.001)$ and high poverty $(P<0.001)$ were regarded as significant risk factors for a poorer survival by univariate analysis (Table 2). Additionally, multivariate analysis was performed by the Cox regression model. The following seven factors were verified as independent prognostic factors for ECC (Table 2), including age ( $\geq 70$, hazard ratio [HR] 1.362, 95\% confidence interval [CI] 1.293-1.436), year of diagnosis (1973-1979, HR 0.839, 95\% CI 0.737-0.955; 1980-1989, HR 0.777, 95\% CI 0.688-0.877; 1990-1999,
HR 0.810, 95\% CI 0.724-0.906; 2000-2009, HR 0.756, 95\% CI 0.666-0.859), pathological grade (poor/ anaplastic, HR $1.480,95 \%$ CI 1.369-1.599), TNM stage (III/ IV, HR $1.135,95 \%$ CI 1.035-1.244), SEER stage (regional, HR 1.157, 95\% CI 1.079-1.241; distant, HR 2.225, 95\% CI 2.034-2.435), socioeconomic status (medium poverty, HR 1.074, 95\% CI 1.004-1.149; high poverty, HR 1.191, 95\% CI 1.094-1.297), and marital status (never married, HR 1.150, 95\% CI 1.061-1.247; divorced/separated, HR 1.183 , 95\% CI 1.087-1.287; widowed, HR 1.179, 95\% CI 1.104-1.260). However, no statistical differences were found with regard to gender and ethnicity according to multivariate survival analysis.

\section{Influence of marital status on ICSS}

Although a better 5-year ICSS was observed in married individuals compared with unmarried individuals diagnosed with ICC (13.0\% vs $11.6 \%$ ) (Figure 1C), the logrank $\chi 2$ test indicated that the difference was marginally significant $(P=0.056)$. After separating the unmarried status based on being never married, divorced/separated, and widowed, a substantial decrease in cancer-specific survival was observed in widowed patients compared to married patients $(7.7 \%$ vs $13.0 \%, P=0.006)$ (Figure 1D). After controlling other covariates using Cox regression model, it showed that the widowed status was an independent prognostic factor for poor survival outcome of ICC patients (HR 1.379, 95\% CI 1.143-1.664, $P=$ 0.001). Compared with married ICC patients, no significant difference was found in never married group (HR 1.003, 95\% CI 0.828-1.214, $P=0.977$ ) and divorced/separated group (HR 1.180, 95\% CI 0.963-1.446, $P=0.111$ ).

In addition, several covariates including gender $(P=0.016)$, age $(P<0.001)$, year of diagnosis $(P<0.001)$, pathological grading $(P<0.001)$, TNM stage $(P<0.001)$, SEER stage $(P<0.001)$ and socioeconomic status $(P<0.001)$ were proved as significant risk factors for ICC prognosis by univariate analysis (Table 2). Multivariate analysis was further carried out to identify the independent predictive factors, as follows: gender (female, HR 0.816, $95 \%$ CI $0.719-0.927)$, age ( $\geq 70$, HR $1.276,95 \%$ CI $1.114-$ 1.461 ), year of diagnosis (1990-1999, HR $0.635,95 \%$ CI 0.423-0.952), pathological grading (poor/anaplastic, HR $1.540,95 \%$ CI 1.282-1.850), TNM stage (III/ IV, HR $1.535,95 \%$ CI $1.217-1.938$ ), SEER stage (regional, HR $1.236,95 \%$ CI 1.025-1.491; distant, HR 1.852, 95\% CI 1.523-2.252), and socioeconomic status (medium poverty, HR 1.232, 95\% CI 1.028-1.478; high poverty, HR 1.335, 95\% CI 1.076-1.657) (Table 2).

\section{Subgroup analysis of marital status on ECSS and ICSS according to SEER stage}

As shown in Table 3 and Figure 2A-2C, we assessed the effects of marital status on ECSS at each SEER stage. 
Table 1: Baseline demographic and cancer characteristics of cholangiocarcinoma patients in SEER database

\begin{tabular}{|c|c|c|c|c|c|c|}
\hline \multirow{2}{*}{ Characteristic } & Total & Married & Never married & $\begin{array}{l}\text { Divorced/ } \\
\text { Separated }\end{array}$ & Widowed & \multirow{2}{*}{$\boldsymbol{P}$} \\
\hline & $\begin{array}{c}(n=10128) \\
\mathrm{N}(\%)\end{array}$ & $\begin{array}{c}(n=5926) \\
N(\%)\end{array}$ & $\begin{array}{c}(n=1150) \\
\mathrm{N}(\%)\end{array}$ & $\begin{array}{c}(n=947) \\
\mathrm{N}(\%)\end{array}$ & $\begin{array}{c}(n=2105) \\
\mathrm{N}(\%)\end{array}$ & \\
\hline Gender & & & & & & $<0.001$ \\
\hline Male & $5265(52.0)$ & $3744(63.2)$ & $629(54.7)$ & $458(48.4)$ & $434(20.6)$ & \\
\hline Female & $4863(48.0)$ & $2182(36.8)$ & $521(45.3)$ & $489(51.6)$ & $1671(79.4)$ & \\
\hline Age & & & & & & $<0.001$ \\
\hline$<70$ & $5059(50.0)$ & $3332(56.2)$ & $782(68.0)$ & $577(60.9)$ & $368(17.5)$ & \\
\hline$\geq 70$ & $5069(50.0)$ & $2594(43.8)$ & $368(32.0)$ & $370(39.1)$ & $1737(82.5)$ & \\
\hline Ethnicity & & & & & & $<0.001$ \\
\hline White & 7969(78.7) & $4725(79.7)$ & $828(72.0)$ & $758(80.0)$ & $1658(78.8)$ & \\
\hline Black & $835(8.2)$ & $337(5.7)$ & $186(16.2)$ & 111(11.7) & $201(9.5)$ & \\
\hline Other* & $1324(13.1)$ & $864(14.6)$ & $136(11.8)$ & $78(8.2)$ & $246(11.7)$ & \\
\hline Year of diagnosis & & & & & & $<0.001$ \\
\hline 1973-1979 & $496(4.9)$ & $341(5.8)$ & $19(1.7)$ & $52(5.5)$ & $84(4.0)$ & \\
\hline 1980-1989 & $785(7.8)$ & $445(7.5)$ & $69(6.0)$ & $80(8.4)$ & 191(9.1) & \\
\hline 1990-1999 & $1149(11.3)$ & $670(11.3)$ & $131(11.4)$ & $87(9.2)$ & 261(12.4) & \\
\hline $2000-2009$ & $5074(50.1)$ & 2911(49.1) & $584(50.8)$ & $468(49.4)$ & $1111(52.8)$ & \\
\hline 2010-2013 & $2624(25.9)$ & $1559(26.3)$ & $347(30.2)$ & $260(27.5)$ & $458(21.8)$ & \\
\hline Pathological grading & & & & & & $<0.001$ \\
\hline Well/Moderate & $2826(27.9)$ & $1839(31.0)$ & $308(26.8)$ & $251(26.5)$ & $428(20.3)$ & \\
\hline Poor/Anaplastic & $1670(16.5)$ & $1044(17.6)$ & $173(15.0)$ & $180(19.0)$ & $273(13.0)$ & \\
\hline Unknown & $5632(55.6)$ & $3043(51.3)$ & $669(58.2)$ & $516(54.5)$ & $1404(66.7)$ & \\
\hline TNM Stage & & & & & & $<0.001$ \\
\hline $\mathrm{I} / \mathrm{II}$ & $2664(26.3)$ & $1614(27.2)$ & $306(26.6)$ & $263(27.8)$ & $481(22.9)$ & \\
\hline III/IV & $2256(22.3)$ & $1357(22.9)$ & $306(26.6)$ & $245(25.9)$ & $348(16.5)$ & \\
\hline Unknown & $5208(51.4)$ & $2955(49.9)$ & $538(46.8)$ & $439(46.4)$ & $1276(60.6)$ & \\
\hline SEER Stage & & & & & & $<0.001$ \\
\hline Localized & $2181(21.5)$ & $1250(21.1)$ & $230(20.0)$ & $202(21.3)$ & $499(23.7)$ & \\
\hline Regional & $3978(39.3)$ & $2504(42.3)$ & $448(39.0)$ & $359(37.9)$ & $667(31.7)$ & \\
\hline Distant & $2445(24.1)$ & $1476(24.9)$ & $299(26.0)$ & $253(26.7)$ & $417(19.8)$ & \\
\hline Unstaged & $1524(15.0)$ & $696(11.7)$ & $173(15.0)$ & $133(14.0)$ & $522(24.8)$ & \\
\hline Socioeconomic Status & & & & & & $<0.001$ \\
\hline Low poverty & $1570(15.5)$ & $966(16.3)$ & $146(12.7)$ & $139(14.7)$ & $319(15.2)$ & \\
\hline Medium poverty & $6868(67.8)$ & $4048(68.3)$ & $806(70.1)$ & $651(68.7)$ & $1363(64.8)$ & \\
\hline High poverty & $1690(16.7)$ & $912(15.4)$ & $198(17.2)$ & $157(16.6)$ & $423(20.1)$ & \\
\hline
\end{tabular}

Abbreviations: SEER, Surveillance, Epidemiology, and End Results.

*Other includes American Indian/Alaska native, Asian/Pacific Islander, and unknown.

Univariate analysis showed that married ECC patients had the highest survival rate among all tumor stages: married patients had a $9.2 \%$ increase in 5-year ECSS compared with widowed patients for localized stage tumors $(22.0 \%$ vs $12.8 \%$ ), a $7.5 \%$ increase for regional stage tumors
(17.5\% vs $10.0 \%)$, and a $0.3 \%$ increase for distant stage tumors $(2.9 \%$ vs $2.6 \%)$. Multivariate Cox regression analyses were carried out for different SEER stages. Marital status was validated as an independent predictor of ECC survival at localized stage (widowed, HR 1.196, 
95\% CI 1.043-1.372), regional stage (never married, HR 1.190, 95\% CI 1.047-1.353; divorced/separated, HR $1.188,95 \%$ CI 1.038-1.361; widowed, HR 1.124, 95\% CI 1.004-1.257), and distant stage (never married, HR 1.237, 95\% CI 1.066-1.435).

In ICC patients, univariate and multivariate analysis demonstrated that widowed patients had a poorer outcome (HR 1.715, 95\% CI 1.204-2.443) compared with married patients at regional stage, and no significant result was detected in any other subgroup (Table 3 and Figure 2D-2F).
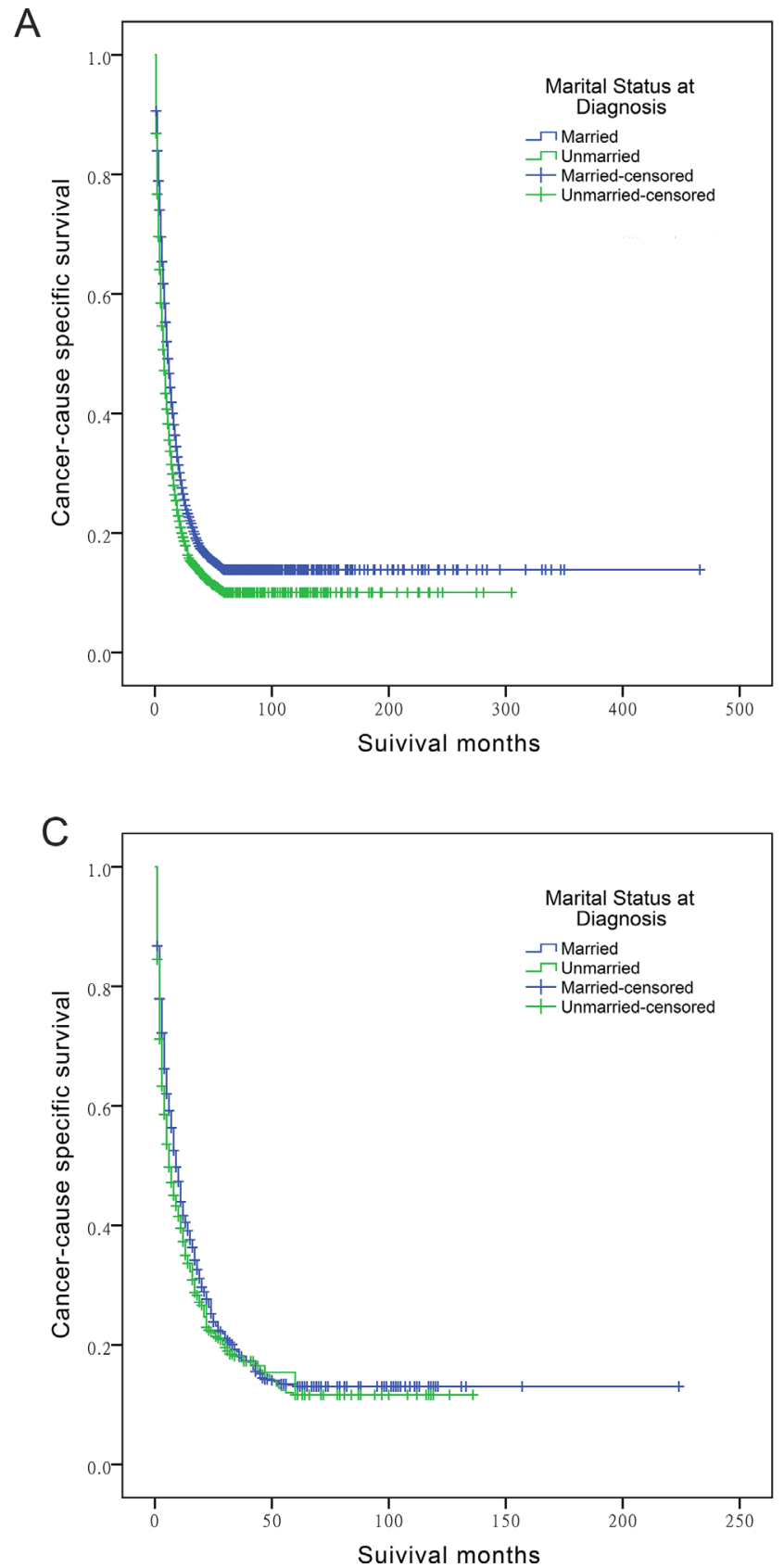

\section{Subgroup analysis of marital status on ECSS and ICSS according to age at diagnosis}

We further examined whether marital status was correlated to 5-year ECSS in patients diagnosed at different ages. Univariate analysis of marital status indicated that married patients had a better 5-year ECSS in all age subgroups (age $<70$, 5-year ECSS 15.9\%, $P<0.001$; age $\geq 70$, 5-year ECSS $11.4 \%, P<0.001)$ (Table 4). Cox regression analysis confirmed the independent prognostic role of marital status in both
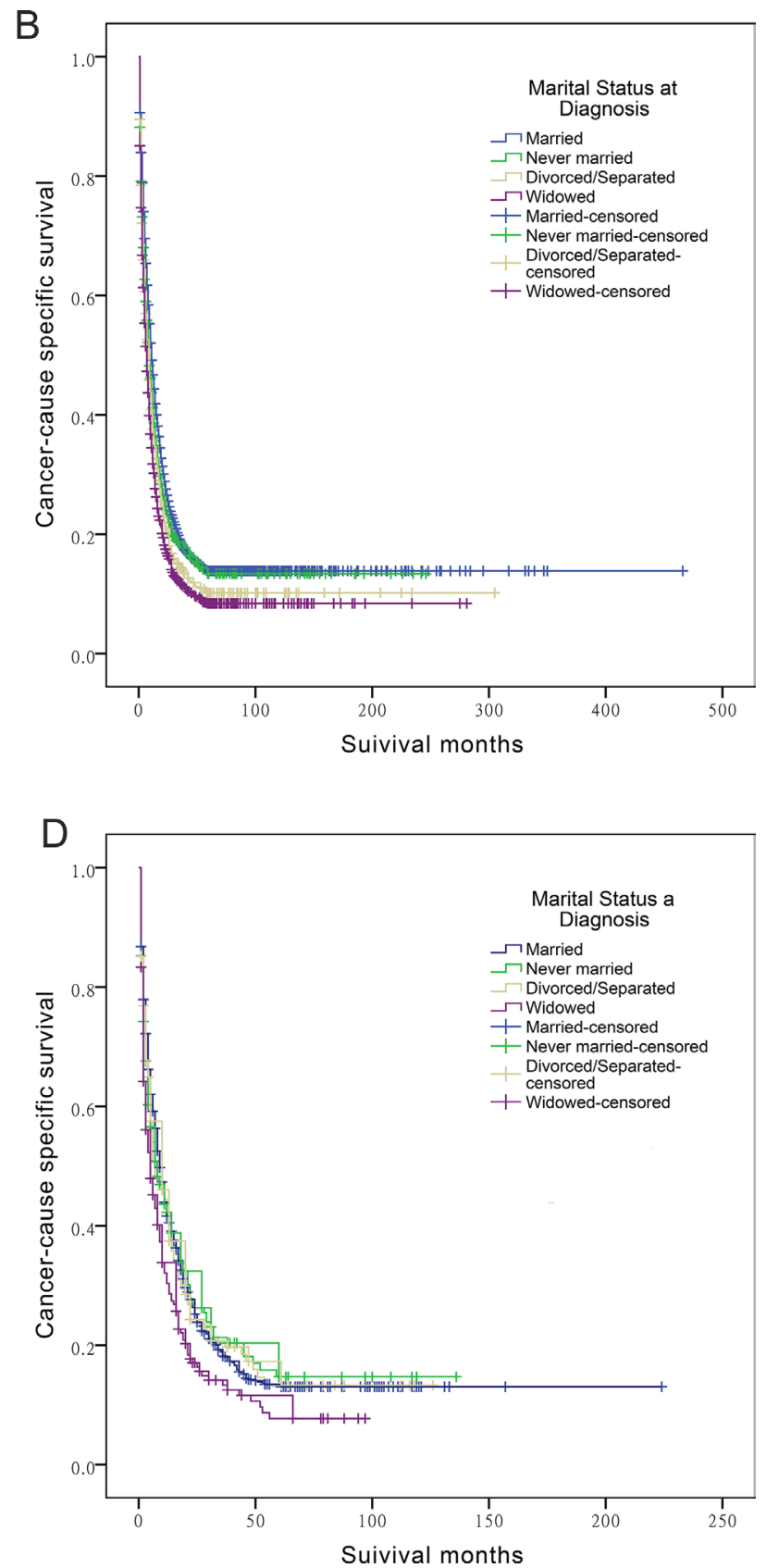

Figure 1: Survival curves in cholangiocarcinoma patients. (A) The survival curve between married and unmarried ECC patients: $\chi^{2}=104.720(P<0.001)$; (B) The survival curve between married, never married, divorced/separated, and widowed ECC patients: $\chi^{2}=138.625(P<0.001)$; (C) The survival curve between married and unmarried ICC patients: $\chi^{2}=3.646(P=0.056)$; (D) The survival curve between married, never married, divorced/separated, and widowed ICC patients: $\chi^{2}=12.351(P=0.006)$. 
Table 2: Univariate and multivariate survival analysis for evaluating the influence of marital status on extrahepatic/intrahepatic cholangiocarcinoma cause-specific survival in SEER database

\begin{tabular}{|c|c|c|c|c|c|c|c|c|c|c|}
\hline \multirow[b]{3}{*}{ Variable } & \multicolumn{5}{|c|}{ Extrahepatic cholangiocarcinoma } & \multicolumn{5}{|c|}{ Intrahepatic cholangiocarcinoma } \\
\hline & \multicolumn{3}{|c|}{ Univariate analysis } & \multicolumn{2}{|c|}{ Multivariate analysis } & \multirow[b]{2}{*}{$\begin{array}{c}\text { 5-year } \\
\text { CCS }\end{array}$} & \multicolumn{2}{|c|}{ Univariate analysis } & \multicolumn{2}{|c|}{ Multivariate analysis } \\
\hline & $\begin{array}{c}\text { 5-year } \\
\text { CCS }\end{array}$ & $\begin{array}{c}\text { Log rank } \\
\chi^{2} \text { test }\end{array}$ & $P$ & HR $(95 \%$ CI $)$ & $P$ & & $\begin{array}{c}\text { Log rank } \\
\chi^{2} \text { test }\end{array}$ & $\boldsymbol{P}$ & HR $(95 \%$ CI $)$ & $P$ \\
\hline Gender & & 57.597 & $<0.001$ & & 0.098 & & 5.841 & 0.016 & & 0.002 \\
\hline Male & $13.7 \%$ & & & Reference & & $11.6 \%$ & & & Reference & \\
\hline Female & $10.8 \%$ & & & $1.044(0.992-1.099)$ & & $13.4 \%$ & & & $0.816(0.719-0.927)$ & \\
\hline Age & & 192.854 & $<0.001$ & & $<0.001$ & & 13.549 & $<0.001$ & & $<0.001$ \\
\hline$<70$ & $15.4 \%$ & & & Reference & & $14.1 \%$ & & & Reference & \\
\hline$\geq 70$ & $9.4 \%$ & & & $1.362(1.293-1.436)$ & & $9.7 \%$ & & & $1.276(1.114-1.461)$ & \\
\hline Ethnicity & & 10.477 & 0.005 & & NI & & 1.563 & 0.458 & & NI \\
\hline White & $12.3 \%$ & & & & & $11.8 \%$ & & & & \\
\hline Black & $10.2 \%$ & & & & & $10.1 \%$ & & & & \\
\hline Other* & $13.4 \%$ & & & & & $21.3 \%$ & & & & \\
\hline Year of diagnosis & & 27.524 & $<0.001$ & & $<0.001$ & & 42.004 & $<0.001$ & & 0.007 \\
\hline $1973-1979$ & $8.8 \%$ & & & Reference & & $4.2 \%$ & & & Reference & \\
\hline 1980-1989 & $10.6 \%$ & & & $0.839(0.737-0.955)$ & 0.008 & $3.7 \%$ & & & $1.090(0.755-1.574)$ & 0.645 \\
\hline 1990-1999 & $13.1 \%$ & & & $0.777(0.688-0.877)$ & $<0.001$ & $10.0 \%$ & & & $0.635(0.423-0.952)$ & 0.028 \\
\hline 2000-2009 & $12.5 \%$ & & & $0.810(0.724-0.906)$ & $<0.001$ & $13.9 \%$ & & & $0.748(0.542-1.031)$ & 0.076 \\
\hline 2010-2013 & \# & & & $0.756(0.666-0.859)$ & $<0.001$ & I & & & $0.813(0.571-1.156)$ & 0.249 \\
\hline Pathological grading & & 622.853 & $<0.001$ & & $<0.001$ & & 90.577 & $<0.001$ & & $<0.001$ \\
\hline Well/ Moderate & $22.5 \%$ & & & Reference & & $22.3 \%$ & & & Reference & \\
\hline Poor/ Anaplastic & $11.9 \%$ & & & $1.480(1.369-1.599)$ & $<0.001$ & $9.4 \%$ & & & $1.540(1.282-1.850)$ & $<0.001$ \\
\hline Unknown & $6.9 \%$ & & & $1.679(1.577-1.787)$ & $<0.001$ & $8.5 \%$ & & & $1.683(1.441-1.966)$ & $<0.001$ \\
\hline TNM Stage & & 407.369 & $<0.001$ & & $<0.001$ & & 92.752 & $<0.001$ & & $<0.001$ \\
\hline $\mathrm{I} / \mathrm{II}$ & $20.1 \%$ & & & Reference & & $30.0 \%$ & & & Reference & \\
\hline III/ IV & $4.4 \%$ & & & $1.135(1.035-1.244)$ & 0.007 & $6.2 \%$ & & & $1.535(1.217-1.938)$ & $<0.001$ \\
\hline Unknown & $11.3 \%$ & & & $1.194(1.104-1.290)$ & $<0.001$ & $9.5 \%$ & & & $1.704(1.371-2.118)$ & $<0.001$ \\
\hline SEER Stage & & 943.197 & $<0.001$ & & $<0.001$ & & 131.019 & $<0.001$ & & $<0.001$ \\
\hline Localized & $18.9 \%$ & & & Reference & & $21.9 \%$ & & & Reference & \\
\hline Regional & $15.6 \%$ & & & $1.157(1.079-1.241)$ & $<0.001$ & $9.5 \%$ & & & $1.236(1.025-1.491)$ & 0.027 \\
\hline Distant & $2.6 \%$ & & & $2.225(2.034-2.435)$ & $<0.001$ & $3.7 \%$ & & & $1.852(1.523-2.252)$ & $<0.001$ \\
\hline Unstaged & $9.5 \%$ & & & $1.208(1.103-1.323)$ & $<0.001$ & $14.6 \%$ & & & $1.107(0.876-1.400)$ & 0.395 \\
\hline Socioeconomic Status & & 17.045 & $<0.001$ & & $<0.001$ & & 9.589 & 0.008 & & 0.027 \\
\hline Low poverty & $12.2 \%$ & & & Reference & & $16.5 \%$ & & & Reference & \\
\hline Medium poverty & $12.7 \%$ & & & $1.074(1.004-1.149)$ & 0.038 & $12.2 \%$ & & & $1.232(1.028-1.478)$ & 0.024 \\
\hline High poverty & $10.4 \%$ & & & $1.191(1.094-1.297)$ & $<0.001$ & $10.5 \%$ & & & $1.335(1.076-1.657)$ & 0.009 \\
\hline Marital Status & & 138.625 & $<0.001$ & & $<0.001$ & & 12.351 & 0.006 & & 0.005 \\
\hline Married & $13.9 \%$ & & & Reference & & $13.0 \%$ & & & Reference & \\
\hline Never married & $13.4 \%$ & & & $1.150(1.061-1.247)$ & 0.001 & $14.7 \%$ & & & $1.003(0.828-1.214)$ & 0.977 \\
\hline $\begin{array}{l}\text { Divorced/ } \\
\text { Separated }\end{array}$ & $10.2 \%$ & & & $1.183(1.087-1.287)$ & $<0.001$ & $13.3 \%$ & & & $1.180(0.963-1.446)$ & 0.111 \\
\hline Widowed & $8.4 \%$ & & & $1.179(1.104-1.260)$ & $<0.001$ & $7.7 \%$ & & & $1.379(1.143-1.664)$ & 0.001 \\
\hline
\end{tabular}

Abbreviations: SEER, Surveillance, Epidemiology, and End Results; CCS, cancer cause-specific survival; HR, hazard ratio; CI, confidence interval; NI, not included in the multivariate survival analysis.

*: Other includes American Indian/Alaska native, Asian/Pacific Islander, and unknown.

\#: Because the record in the SEER database ended in 2013, the 5-year CCS of this group did not exist. 

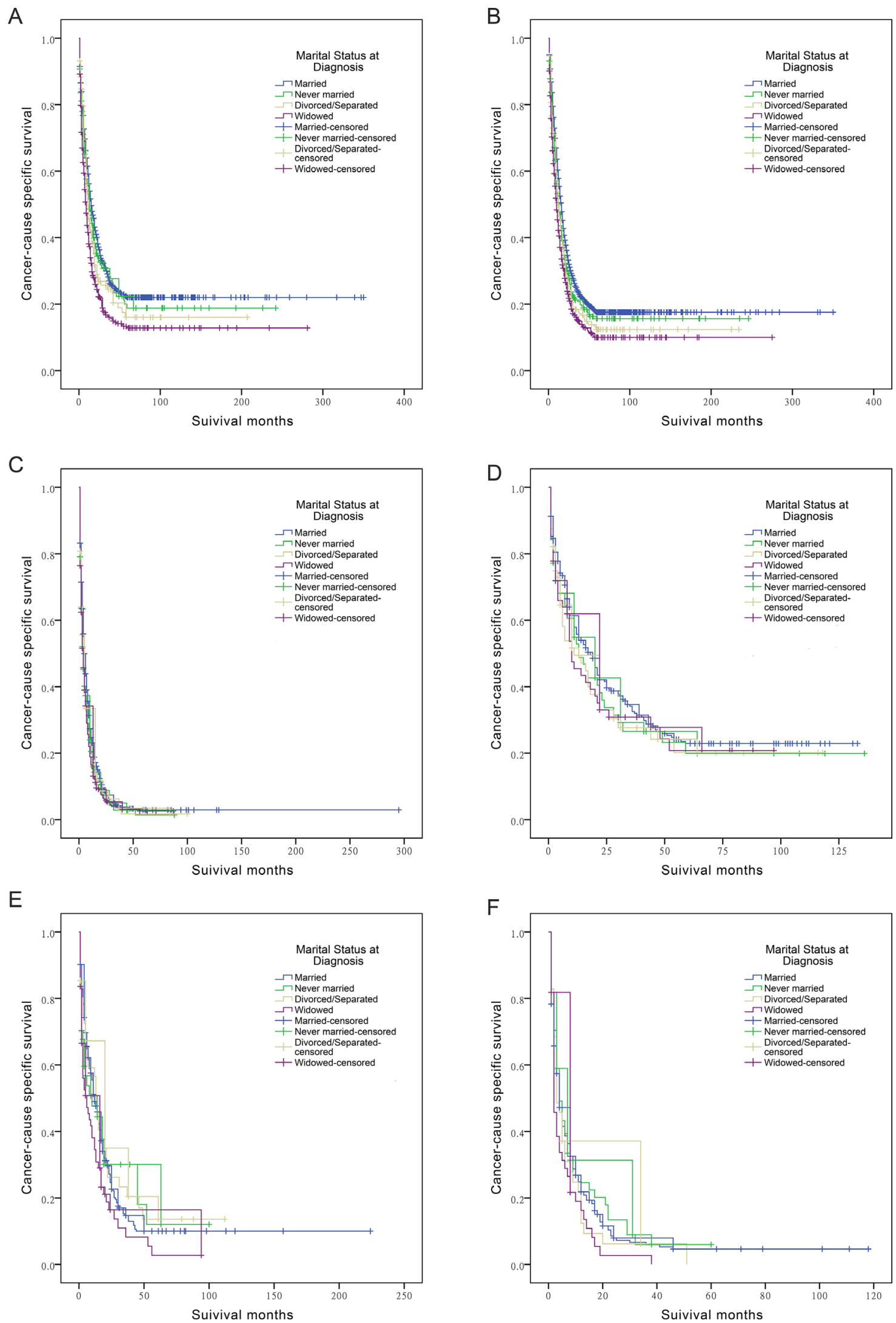

Figure 2: Survival curves in cholangiocarcinoma patients according to marital status. (A) ECC patients at SEER localized stage: $\chi^{2}=39.758(P<0.001)$; (B) ECC patients at SEER regional stage: $\chi^{2}=54.452(P<0.001)$; (C) ECC patients at SEER distant stage: $\chi^{2}=17.9(P<0.001)$; (D) ICC patients at SEER localized stage: $\chi^{2}=2.05(P=0.56)$; (E) ICC patients at SEER regional stage: $\chi^{2}=6.725$ $(P=0.081) ;($ F $)$ ICC patients at SEER distant stage: $\chi^{2}=5.054(P=0.168)$. 
Table 3: Univariate and multivariate survival analysis of marital status on extrahepatic/intrahepatic cholangiocarcinoma cause-specific survival based on different SEER stages

\begin{tabular}{|c|c|c|c|c|c|}
\hline \multirow{2}{*}{ Variable } & \multirow[b]{2}{*}{ 5-year CCS } & \multicolumn{2}{|c|}{ Univariate analysis } & \multicolumn{2}{|c|}{ Multivariate analysis } \\
\hline & & Log rank $\chi^{2}$ test & $P$ & HR $(95 \% \mathrm{CI})$ & $\boldsymbol{P}$ \\
\hline \multicolumn{6}{|c|}{ Extrahepatic cholangiocarcinoma } \\
\hline \multicolumn{6}{|c|}{ SEER stage } \\
\hline \multicolumn{6}{|l|}{ Localized } \\
\hline Marital Status & & 39.758 & $<0.001$ & & 0.034 \\
\hline Married & $22.0 \%$ & & & Reference & \\
\hline Never married & $18.8 \%$ & & & $1.165(0.954-1.423)$ & 0.133 \\
\hline Divorced/Separated & $16.0 \%$ & & & $1.191(0.967-1.468)$ & 0.1 \\
\hline Widowed & $12.8 \%$ & & & $1.196(1.043-1.372)$ & 0.01 \\
\hline \multicolumn{6}{|l|}{ Regional } \\
\hline Marital Status & & 54.452 & $<0.001$ & & 0.004 \\
\hline Married & $17.5 \%$ & & & Reference & \\
\hline Never married & $15.6 \%$ & & & $1.190(1.047-1.353)$ & 0.008 \\
\hline Divorced/Separated & $12.3 \%$ & & & $1.188(1.038-1.361)$ & 0.013 \\
\hline Widowed & $10.0 \%$ & & & $1.124(1.004-1.257)$ & 0.042 \\
\hline \multicolumn{6}{|l|}{ Distant } \\
\hline Marital Status & & 17.9 & $<0.001$ & & 0.008 \\
\hline Married & $2.9 \%$ & & & Reference & \\
\hline Never married & $1.4 \%$ & & & $1.237(1.066-1.435)$ & 0.005 \\
\hline Divorced/Separated & $1.7 \%$ & & & $1.162(1.000-1.351)$ & 0.05 \\
\hline Widowed & $2.6 \%$ & & & $1.134(0.997-1.289)$ & 0.056 \\
\hline \multicolumn{6}{|c|}{ Intrahepatic cholangiocarcinoma } \\
\hline \multicolumn{6}{|c|}{ SEER stage } \\
\hline \multicolumn{6}{|l|}{ Localized } \\
\hline Marital Status & & 2.06 & 0.56 & & NI \\
\hline Married & $22.9 \%$ & & & & \\
\hline Never married & $19.9 \%$ & & & & \\
\hline Divorced/Separated & $20.2 \%$ & & & & \\
\hline Widowed & $20.8 \%$ & & & & \\
\hline \multicolumn{6}{|l|}{ Regional } \\
\hline Marital Status & & 6.725 & 0.081 & & 0.023 \\
\hline Married & $10.0 \%$ & & & Reference & \\
\hline Never married & $12.0 \%$ & & & $0.960(0.639-1.441)$ & 0.843 \\
\hline Divorced/Separated & $13.6 \%$ & & & $1.070(0.727-1.574)$ & 0.731 \\
\hline Widowed & $2.7 \%$ & & & $1.715(1.204-2.443)$ & 0.003 \\
\hline \multicolumn{6}{|l|}{ Distant } \\
\hline Marital Status & & 5.054 & 0.168 & & NI \\
\hline Married & $4.6 \%$ & & & & \\
\hline Never married & $6.0 \%$ & & & & \\
\hline Divorced/Separated & $0.0 \%$ & & & & \\
\hline Widowed & $0.0 \%$ & & & & \\
\hline
\end{tabular}

Abbreviations: SEER, Surveillance, Epidemiology, and End Results; CCS, cancer cause-specific survival; HR, hazard ratio; $\mathrm{CI}$, confidence interval; NI, not included in the multivariate survival analysis. 
younger patients (divorced/separated, HR 1.185, 95\% CI 1.060-1.325) and older patients (never married, HR 1.258, 95\% CI 1.104-1.434; divorced/separated HR 1.198, 95\% CI 1.054-1.362; widowed HR 1.212, 95\% CI 1.128-1.302) (Table 4 and Figure 3A-3B).

Univariate and multivariate survival analysis showed that widowed patients suffered from poorer survival outcomes (HR 1.305, 95\% CI 1.029-1.654) compared to married patients when diagnosed at older age. In addition, there was no significant result observed in age $<70$ subgroup (Table 4 and Figure 3C-3D).
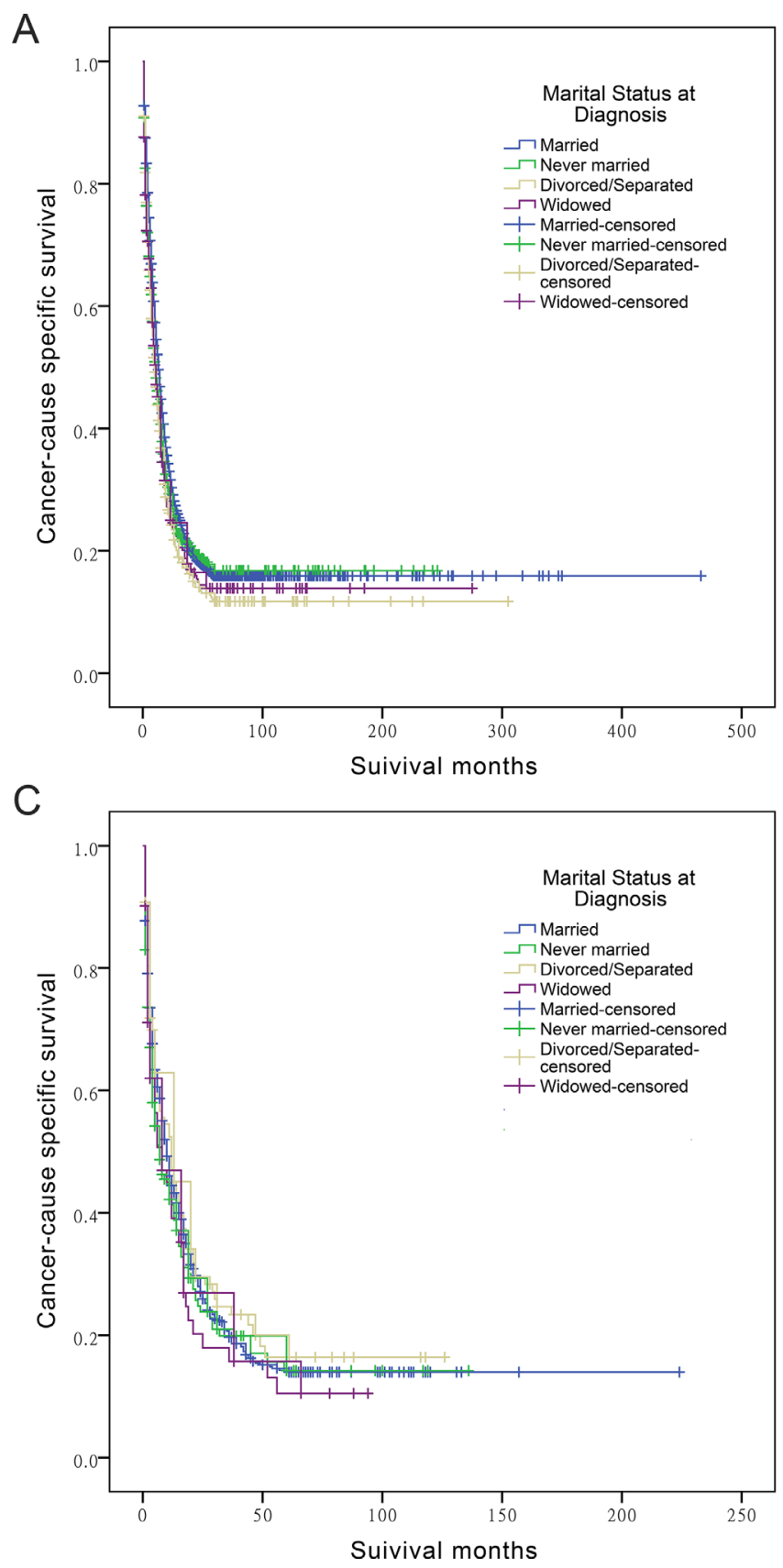

\section{DISCUSSION}

To the best of our knowledge, the current study is the first study to date comprehensively investigating the influence of marital status on the survival of patients with cholangiocarcinoma. We found a higher risk of death associated with being unmarried, especially widowed. Further subgroup analysis on SEER stage and age verified the prognostic value of marital status in cholangiocarcinoma.

Our results demonstrated that widowed cholangiocarcinoma patients were more likely to suffer
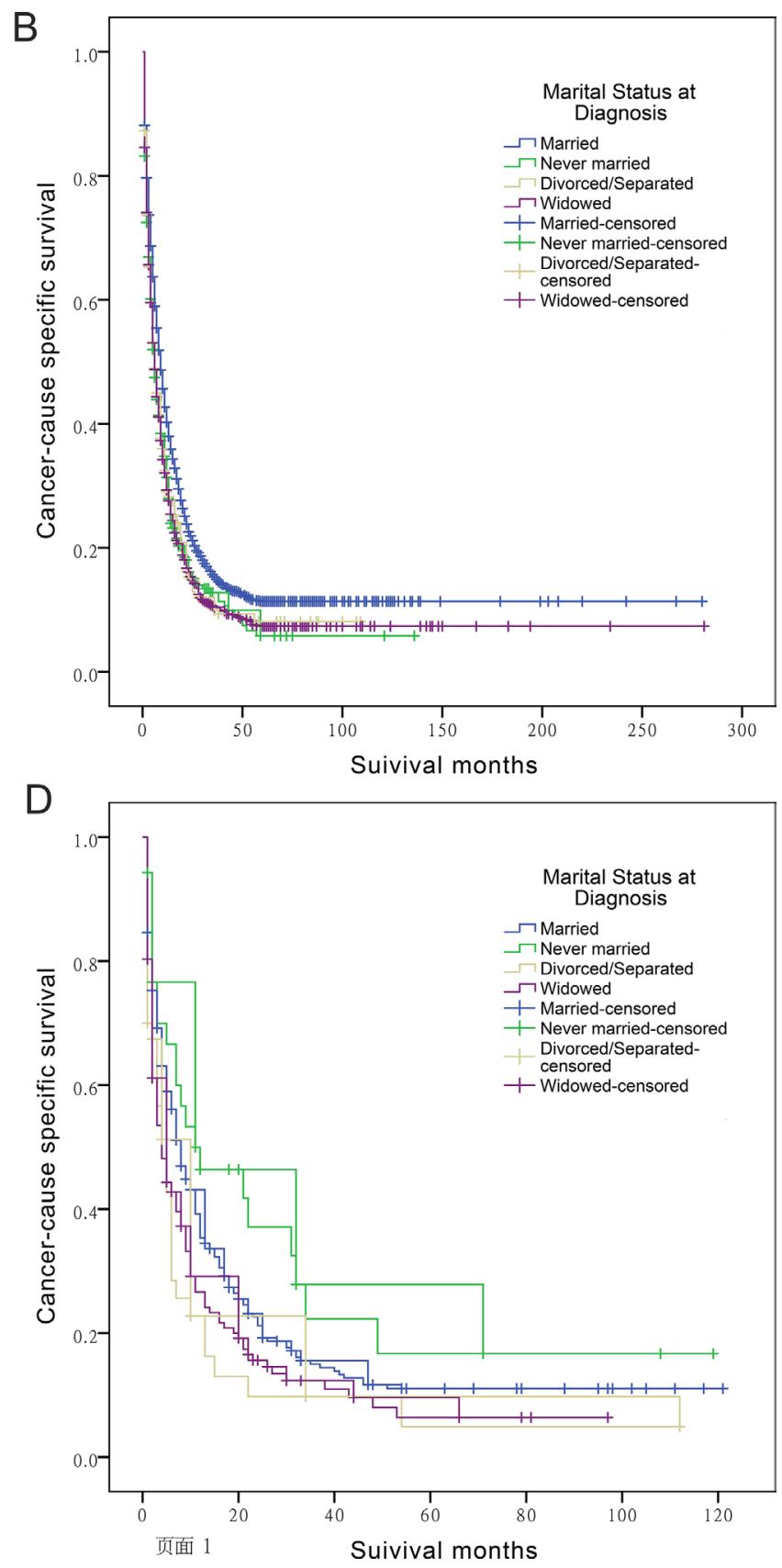

Figure 3: Survival curves in cholangiocarcinoma patients according to marital status. (A) ECC patients aged less than 70: $\chi^{2}=17.994(P<0.001)$; (B) ECC patients aged more than 70: $\chi^{2}=57.184(P<0.001)$; (C) ICC patients aged less than $70: \chi^{2}=2.568$ $(P=0.463)$; (D) ICC patients aged more than 70: $\chi^{2}=13.195(P=0.004)$. 
Table 4: Univariate and multivariate survival analysis of marital status on extrahepatic/intrahepatic cholangiocarcinoma cause-specific survival based on different age groups

\begin{tabular}{|c|c|c|c|c|c|}
\hline \multirow{2}{*}{ Variable } & \multirow[b]{2}{*}{ 5-year CCS } & \multicolumn{2}{|c|}{ Univariate analysis } & \multicolumn{2}{|c|}{ Multivariate analysis } \\
\hline & & Log rank $\chi^{2}$ test & $P$ & $\operatorname{HR}(95 \% \mathrm{CI})$ & $P$ \\
\hline \multicolumn{6}{|c|}{ Extrahepatic cholangiocarcinoma } \\
\hline \multicolumn{6}{|c|}{ Age $<70$} \\
\hline Marital Status & & 17.994 & $<0.001$ & & 0.007 \\
\hline Married & $15.9 \%$ & & & Reference & \\
\hline Never married & $16.8 \%$ & & & $1.107(0.999-1.228)$ & 0.053 \\
\hline Divorced/Separated & $11.7 \%$ & & & $1.185(1.060-1.325)$ & 0.003 \\
\hline Widowed & $13.9 \%$ & & & $1.115(0.971-1.280)$ & 0.124 \\
\hline \multicolumn{6}{|l|}{ Age $\geq 70$} \\
\hline Marital Status & & 57.184 & $<0.001$ & & $<0.001$ \\
\hline Married & $11.4 \%$ & & & Reference & \\
\hline Never married & $5.8 \%$ & & & $1.258(1.104-1.434)$ & 0.001 \\
\hline Divorced/Separated & $8.1 \%$ & & & $1.198(1.054-1.362)$ & 0.006 \\
\hline Widowed & $7.3 \%$ & & & $1.212(1.128-1.302)$ & $<0.001$ \\
\hline \multicolumn{6}{|c|}{ Intrahepatic cholangiocarcinoma } \\
\hline Age $<70$ & & & & & NI \\
\hline Marital Status & & 2.568 & 0.463 & & \\
\hline Married & $14.0 \%$ & & & & \\
\hline Never married & $14.2 \%$ & & & & \\
\hline Divorced/Separated & $16.4 \%$ & & & & \\
\hline Widowed & $10.5 \%$ & & & & \\
\hline \multicolumn{6}{|l|}{ Age $\geq 70$} \\
\hline Marital Status & & 13.195 & 0.004 & & 0.019 \\
\hline Married & $11.0 \%$ & & & Reference & \\
\hline Never married & $16.7 \%$ & & & $0.734(0.473-1.141)$ & 0.169 \\
\hline Divorced/Separated & $4.9 \%$ & & & $1.339(0.925-1.938)$ & 0.122 \\
\hline Widowed & $6.4 \%$ & & & $1.305(1.029-1.654)$ & 0.028 \\
\hline
\end{tabular}

Abbreviations: CCS, cancer cause-specific survival; HR, hazard ratio; CI, confidence interval; NI, not included in the multivariate survival analysis.

from survival disadvantages. Marriage itself may increase the probability of early diagnosis. On most occasions, married patients experience more emotional and social support compared to those who are unmarried [19]. Married individuals are more likely to pursue immediate and aggressive treatment [20]. Besides, constant support provided by a spouse encourages the patients to comply with the prescribed treatment regiments [21, 22]. An alternative hypothesis is that although marriage itself may not necessarily protect the patients, once one has got married, becoming widowed is rather hard to endure both physically and psychologically. In consistent with this standpoint, no significant difference was found in our study between married patients and never married patients in ICC (HR 1.003, 95\% CI 0.828-1.214, $P=0.977$ ).
Widowed patients, by contrast, had a poor survival outcome compared with married patients in ICC (HR $1.379,95 \%$ CI $1.143-1.664, P=0.001)$. It is conceivable that patients who are unmarried, especially the widowed ones, have a more fragile support network. The chronic stress that results from a lack of social support may impact cancer growth and metastasis by neuroendocrine mediators and cytokines [23]. A lack of psychological support leads to decreased activity of natural killer cells and increased mortality in cancer patients [24, 25].

An interesting observation was that the independent predictive value of marital status was more obvious in ECC patients than in those diagnosed with ICC. The never married, divorced/separated, and widowed patients all had poorer survival outcome than married patients in ECC, 
while only the widowed status independently predicted poor ICC survival. Subgroup analysis according to SEER stage and age indicated similar observations. These results suggested that the long-term prognosis of these two subtypes of cholangiocarcinoma was different, further confirming the heterogeneity between ECC and ICC.

In spite of our efforts to make an accurate and comprehensive analysis, some limitations of our analysis need to be addressed. First of all, the retrospective nature of the current study might result in bias and affect the results. Second, the SEER dataset only provides the marital status at diagnosis. Due to insufficient data, we could not describe or analyze information regarding changes in marital status, duration of the marriage, length of being single or the quality of marriage. Finally, cholangiocarcinoma predisposing factors (eg. primary sclerosing cholangitis, choledochal cysts, bile duct stones, inflammatory bowel disease, viral hepatitis, or infections with the liver fluke) were not provided in the SEER database. These confounding factors may potentially affect the results.

Despite these potential limitations, the present study was based on a large population and multiple centers, and is therefore reliable and convincing. We separated unmarried participants based on being never married, divorced/separated, and widowed, and each SEER stage and age group was individually investigated with a variety of traditional risk factors taken into consideration. Our results confirmed the independent prognostic effect of the unmarried status with a varied risk compared to the married status. Furthermore, the results demonstrated that the unmarried patients were heterogeneous, and widowed patients tended to be at the highest risk of cancer causespecific death compared to those in other groups. Social support, psychological factors and advanced tumor stage might be the reasons for survival disadvantages in widowed patients. More social care and support should be provided for the unmarried patients, especially the widowed ones.

\section{MATERIALS AND METHODS}

\section{Patient selection in the SEER dataset}

All primary data were obtained by using SEER*Stat software version 8.3.2 and the SEER database released in April 2016. The SEER dataset contains no identifiers, and has been widely used for studies examining the association between marital status and cancer survival [26-31]. County-level socioeconomic information for the year 2000 was obtained from US Census 2000 files, which was made available by the US Census Bureau and linked to the SEER database.

ECCs were identified by the topography code C24.0 for extrahepatic bile duct with the following morphology codes: 8010, 8020, 8041, 8070, 8140, 8144, 8160, 8161, $8260,8310,8480,8490,8560$ and 8162 . ICCs were defined by topography code C22.0 for liver and morphology codes
8160 and 8161 , or by topography code C22.1 (intrahepatic bile duct) and morphology codes 8010, 8020, 8140, 8160 and 8161 , as previously reported [32].

The exclusion criteria included: (1) patients with ECC or ICC identified only by autopsy or death certificate, (2) patients had more than one primary tumor but ECC/ICC was not the first one, (3) age at diagnosis was less than 18 , (4) unknown survival time or survival time of 0 months, (5) unknown marital status, (6) SEER stage in situ or unknown.

The TNM stage was established according to the criteria described in the American Joint Committee on Cancer (AJCC) Cancer Staging Manual (the $6^{\text {th }}$ edition). Socioeconomic status was determined by the county poverty rate[33, 34], which was defined as the percentage of people in the county living below the national poverty threshold in the 2000 US Census. As previously reported, the county poverty rates were categorized into three levels using the same cut points used in the National Cancer Institute monograph: $<10 \%$ (low-poverty areas), $10 \%-19.99 \%$ (medium-poverty areas), and $\geq 20 \%$ (highpoverty areas) [35].

\section{Statistical analysis}

Differences in baseline characteristics were compared by Pearson chi-squared test for categorical variables. ECSS and ICSS were calculated by the KaplanMeier method and log-rank $\chi 2$ tests were conducted to compare differences between subgroups of each variable. Multivariate Cox proportional hazard models were adopted to determine risk factors that might affect survivorship. All $P$ values were two-sided, and $P<0.05$ was regarded as statistically significant. All data were analyzed by SPSS version 21.0 (Statistics Package for Social Science, Chicago, IL).

\section{Abbreviations}

ECC: extrahepatic cholangiocarcinoma; ICC: intrahepatic cholangiocarcinoma; SEER: Surveillance, Epidemiology, and End Results; HCC: hepatocellular carcinoma; ECSS: extrahepatic cholangiocarcinoma causespecific survival; ICSS: intrahepatic cholangiocarcinoma cause-specific survival; HR: hazard ratio; CI: confidence interval; AJCC: American Joint Committee on Cancer.

\section{ACKNOWLEDGMENTS AND FUNDING}

This work was supported by grants from National Natural Science Foundation of China (Grant no. 81300363, 81521004, 81270553) and Jiangsu Youth Medical Talents (QNRC2016580).

\section{CONFLICTS OF INTEREST}

The authors declare no conflicts of interest. 


\section{REFERENCES}

1. Fitzmaurice C, Dicker D, Pain A, Hamavid H, MoradiLakeh M, MacIntyre MF, Allen C, Hansen G, Woodbrook R, Wolfe C, Hamadeh RR, Moore A, Werdecker A, et al. The Global Burden of Cancer 2013. JAMA Oncol. 2015; 1:505-27. doi: 10.1001/jamaoncol.2015.0735.

2. Banales JM, Cardinale V, Carpino G, Marzioni M, Andersen JB, Invernizzi $\mathrm{P}$, Lind GE, Folseraas $\mathrm{T}$, Forbes SJ, Fouassier L, Geier A, Calvisi DF, Mertens JC, et al. Expert consensus document: Cholangiocarcinoma: current knowledge and future perspectives consensus statement from the European Network for the Study of Cholangiocarcinoma (ENS-CCA). Nat Rev Gastroenterol Hepatol. 2016; 13:261-80. doi: 10.1038/nrgastro.2016.51.

3. Blechacz B, Komuta M, Roskams T, Gores GJ. Clinical diagnosis and staging of cholangiocarcinoma. Nat Rev Gastroenterol Hepatol. 2011; 8:512-22. doi: 10.1038/ nrgastro.2011.131.

4. Saha SK, Zhu AX, Fuchs CS, Brooks GA. Forty-Year Trends in Cholangiocarcinoma Incidence in the U.S.: Intrahepatic Disease on the Rise. Oncologist. 2016; $21: 594$ 9. doi: 10.1634/theoncologist.2015-0446.

5. Wadhwa V, Jobanputra Y, Thota PN, Narayanan Menon KV, Parsi MA, Sanaka MR. Healthcare utilization and costs associated with cholangiocarcinoma. Gastroenterol Rep (Oxf). 2016. doi: 10.1093/gastro/gow026.

6. Yao KJ, Jabbour S, Parekh N, Lin Y, Moss RA. Increasing mortality in the United States from cholangiocarcinoma: an analysis of the National Center for Health Statistics Database. BMC Gastroenterol. 2016; 16:117. doi: 10.1186/ s12876-016-0527-z.

7. Patel T. Increasing incidence and mortality of primary intrahepatic cholangiocarcinoma in the United States. Hepatology. 2001; 33:1353-7. doi: 10.1053/jhep.2001.25087.

8. Wang XD, Qian JJ, Bai DS, Li ZN, Jiang GQ, Yao J. Marital status independently predicts pancreatic cancer survival in patients treated with surgical resection: an analysis of the SEER database. Oncotarget. 2016; 7:24880-7. doi: 10.18632/ oncotarget.8467.

9. Li J, Wang Y, Han F, Wang Z, Xu L, Tong J. Disadvantage of survival outcomes in widowed patients with colorectal neuroendocrine neoplasm: an analysis of surveillance, epidemiology and end results database. Oncotarget. 2016; 7:83200-83207. doi: 10.18632/oncotarget.13078.

10. Wu C, Chen P, Qian JJ, Jin SJ, Yao J, Wang XD, Bai DS, Jiang GQ. Effect of marital status on the survival of patients with hepatocellular carcinoma treated with surgical resection: an analysis of 13,408 patients in the surveillance, epidemiology, and end results (SEER) database. Oncotarget. 2016; 7:79442-79452. doi: 10.18632/oncotarget.12722.

11. He XK, Lin ZH, Qian Y, Xia D, Jin P, Sun LM. Marital status and survival in patients with primary liver cancer. Oncotarget. 2016. doi: 10.18632/oncotarget.11066. [Epub ahead of print].
12. Songserm N, Woradet S, Bureelerd O, Charoenbut P. Evaluation of Cholangiocarcinoma Risk and its Related Factors in Wetland Geographical Communities of Ubon Ratchathani, Thailand. Asian Pac J Cancer Prev. 2016; 17:1811-5. doi: 10.7314/APJCP.2016.17.4.1811.

13. Roskams T. Liver stem cells and their implication in hepatocellular and cholangiocarcinoma. Oncogene. 2006; 25:3818-22. doi: 10.1038/sj.onc.1209558.

14. Cardinale V, Semeraro R, Torrice A, Gatto M, Napoli C, Bragazzi MC, Gentile R, Alvaro D. Intra-hepatic and extrahepatic cholangiocarcinoma: New insight into epidemiology and risk factors. World J Gastrointest Oncol. 2010; 2:40716. doi: 10.4251/wjgo.v2.i11.407.

15. Tyson GL, El-Serag HB. Risk factors for cholangiocarcinoma. Hepatology. 2011; 54:173-84. doi: 10.1002/hep.24351.

16. Palmer WC, Patel T. Are common factors involved in the pathogenesis of primary liver cancers? A meta-analysis of risk factors for intrahepatic cholangiocarcinoma. J Hepatol. 2012; 57:69-76. doi: 10.1016/j.jhep.2012.02.022.

17. Hirschfield GM, Karlsen TH, Lindor KD, Adams DH. Primary sclerosing cholangitis. Lancet. 2013; 382:1587-99. doi: 10.1016/S0140-6736(13)60096-3.

18. Marcano-Bonilla L, Mohamed EA, Mounajjed T, Roberts LR. Biliary tract cancers: epidemiology, molecular pathogenesis and genetic risk associations. Chin Clin Oncol. 2016; 5:61. doi: 10.21037/cco.2016.10.09.

19. Goldzweig G, Andritsch E, Hubert A, Brenner B, Walach N, Perry S, Baider L. Psychological distress among male patients and male spouses: what do oncologists need to know? Ann Oncol. 2010; 21:877-83. doi: 10.1093/annonc/mdp398.

20. Iwashyna TJ, Christakis NA. Marriage, widowhood, and health-care use. Soc Sci Med. 2003; 57:2137-47. doi: 10.1016/S0277-9536(02)00546-4.

21. Aizer AA, Paly JJ, Zietman AL, Nguyen PL, Beard CJ, Rao SK, Kaplan ID, Niemierko A, Hirsch MS, Wu CL, Olumi AF, Michaelson MD, D'Amico AV, et al. Multidisciplinary care and pursuit of active surveillance in low-risk prostate cancer. J Clin Oncol. 2012; 30:3071-6. doi: 10.1200/JCO.2012.42.8466.

22. Cohen SD, Sharma T, Acquaviva K, Peterson RA, Patel SS, Kimmel PL. Social support and chronic kidney disease: an update. Adv Chronic Kidney Dis. 2007; 14:335-44. doi: 10.1053/j.ackd.2007.04.007.

23. Moreno-Smith M, Lutgendorf SK, Sood AK. Impact of stress on cancer metastasis. Future Oncol. 2010; 6:1863-81. doi: $10.2217 /$ fon. 10.142 .

24. Levy SM, Herberman RB, Whiteside T, Sanzo K, Lee J, Kirkwood J. Perceived social support and tumor estrogen/ progesterone receptor status as predictors of natural killer cell activity in breast cancer patients. Psychosom Med. 1990; 52:73-85. doi: 10.1097/00006842-199001000-00006.

25. Schneider S, Moyer A. Depression as a predictor of disease progression and mortality in cancer patients: a metaanalysis. Cancer. 2010; 116:3304; author reply -5. doi: 10.1002/cncr.25318. 
26. Aizer AA, Chen MH, McCarthy EP, Mendu ML, Koo S, Wilhite TJ, Graham PL, Choueiri TK, Hoffman KE, Martin NE, Hu JC, Nguyen PL. Marital status and survival in patients with cancer. J Clin Oncol. 2013; 31:3869-76. doi: 10.1200/JCO.2013.49.6489.

27. Costa LJ, Brill IK, Brown EE. Impact of marital status, insurance status, income, and race/ethnicity on the survival of younger patients diagnosed with multiple myeloma in the United States. Cancer. 2016; 122:3183-90. doi: 10.1002/ cncr.30183.

28. Lowery WJ, Stany MP, Phippen NT, Bunch KP, Oliver KE, Tian C, Maxwell GL, Darcy KM, Hamilton CA. Survival advantage of marriage in uterine cancer patients contrasts poor outcome for widows: a Surveillance, Epidemiology and End Results study. Gynecol Oncol. 2015; 136:328-35. doi: 10.1016/j.ygyno.2014.12.027.

29. Inverso G, Mahal BA, Aizer AA, Donoff RB, Chau NG, Haddad RI. Marital status and head and neck cancer outcomes. Cancer. 2015; 121:1273-8. doi: 10.1002/ cncr.29171.

30. Alamanda VK, Song Y, Holt GE. Effect of marital status on treatment and survival of extremity soft tissue sarcoma. Ann Oncol. 2014; 25:725-9. doi: 10.1093/annonc/mdt583.

31. Li M, Dai CY, Wang YN, Chen T, Wang L, Yang P, Xie D, Mao R, Chen C. Marital status is an independent prognostic factor for tracheal cancer patients: an analysis of the SEER database. Oncotarget. 2016; 7:77152-77162. doi: 10.18632/ oncotarget.12809.

32. Welzel TM, Graubard BI, El-Serag HB, Shaib YH, Hsing AW, Davila JA, McGlynn KA. Risk factors for intrahepatic and extrahepatic cholangiocarcinoma in the United States: a population-based case-control study. Clin Gastroenterol Hepatol. 2007; 5:1221-8. doi: 10.1016/j. cgh.2007.05.020.

33. Krieger N, Chen JT, Waterman PD, Rehkopf DH, Subramanian SV. Race/ethnicity, gender, and monitoring socioeconomic gradients in health: a comparison of areabased socioeconomic measures--the public health disparities geocoding project. Am J Public Health. 2003; 93:1655-71. doi: 10.2105/AJPH.93.10.1655.

34. Krieger N, Chen JT, Waterman PD, Soobader MJ, Subramanian SV, Carson R. Geocoding and monitoring of US socioeconomic inequalities in mortality and cancer incidence: does the choice of area-based measure and geographic level matter?: the Public Health Disparities Geocoding Project. Am J Epidemiol. 2002; 156:471-82. doi: 10.1093/aje/kwf068.

35. Sun H, Ma H, Hong G, Sun H, Wang J. Survival improvement in patients with pancreatic cancer by decade: a period analysis of the SEER database, 1981-2010. Sci Rep. 2014; 4:6747. doi: 10.1038/srep06747. 